Supporting information 


\section{Bacteria-Triggered pH-Responsive Osteopotentiating Coating on \\ 3D-Printed Polyetheretherketone Scaffolds for Infective Bone Defect}

\section{Repair}

Yi Deng ${ }^{\dagger, \dagger}$, Xiuyuan $\mathrm{Shi}^{\#}$, Yong Chen ${ }^{\dagger}$, Weizhong Yang ${ }^{\dagger, *}$, Yuan $\mathrm{Ma}^{\text {f,* }}$, Xiao-Lei Shi ${ }^{\perp}$, Pingan Song ${ }^{\perp}$, Matthew S. Darguschg ${ }^{\wedge}$, Zhi-Gang Chen ${ }^{\perp, *}$

${ }^{\dagger}$ College of Materials Science and Engineering, School of Chemical Engineering,

State Key Laboratory of Polymer Materials Engineering, Sichuan University, Chengdu 610065, China

Department of Mechanical Engineering, The University of Hong Kong, Hong Kong SAR 999077, China

\#Department of Materials, Imperial College London, South Kensington Campus, London SW7 2AZ, UK

${ }^{\int}$ Department of Neurosurgery, The General Hospital of Western Theater Command, Chengdu 610083, China

${ }^{\perp}$ Centre for Future Materials, University of Southern Queensland, Springfield Central, Queensland 4300, Australia

`Centre for Materials Processing and Manufacturing, University of Queensland, Brisbane, Queensland 4072, Australia 


\section{EXPERIMENTAL SECTION}

\section{D Printing of Porous PEEK Scaffolds}

Pure PEEK wires $\left(\rho=1.30 \mathrm{~g} / \mathrm{cm}^{3}\right.$, Victrex, UK) were used as the source materials in order to fabricate cylindrical PEEK scaffolds $(\Phi=8 \mathrm{~mm}$ and $H=4 \mathrm{~mm})$ with an open porous framework via fused deposition modeling (FDM). A 3D model of objects was prepared using Solidworks software and converted to STL data that controlled our home-made 3D printing machine. After being melted at $390{ }^{\circ} \mathrm{C}$, the printable PEEK wires were injected into printing heads. The printing parameters are listed (nozzle diameter: $0.4 \mathrm{~mm}$; printing speed: $40 \mathrm{~mm} / \mathrm{s}$; plate temperature: $160{ }^{\circ} \mathrm{C}$; infill percentage: $100 \%$ ). The printer head moved back and forth repeatedly during the process of 3D printing, yielding interconnecting mesh networks in a layer-by-layer fashion within about 2 hours for printing all scaffolds. After that, the thus-printed PEEK scaffolds were undergone an air blowing and ultrasonic cleaning in acetone, alcohol, and deionized (D.I.) water repeatedly to detach impurities and were denoted as $3 \mathrm{P}$.

\section{Surface Biofunctionalization}

The 1.5X SBF (Table S2) was firstly prepared by dissolving these chemical reagents including $\mathrm{NaCl}, \mathrm{NaHCO}_{3}, \mathrm{KCl}, \mathrm{K}_{2} \mathrm{HPO}_{4} \cdot 3 \mathrm{H}_{2} \mathrm{O}, \mathrm{MgCl}_{2} \cdot 6 \mathrm{H}_{2} \mathrm{O}, \mathrm{CaCl}_{2}$, and $\mathrm{Na}_{2} \mathrm{SO}_{4}$ using D.I. water in a specific sequence according to the previous work. ${ }^{1,2}$ Then the solution was finally buffered to $\mathrm{pH}=7.4$ using $\left(\mathrm{CH}_{2} \mathrm{OH}\right)_{3} \mathrm{CNH}_{2}$ and $1 \mathrm{~mol} / \mathrm{L} \mathrm{HCl}$ solution under $37^{\circ} \mathrm{C}$. All reagents were purchased from Chengdu KeLong Reagent.

To produce two types of multilayer Ag/apatite biofunctionalized scaffolds, we 
applied a two-step pDA functionalization approach: (1) 3P-Ag-AP scaffolds: Firstly, the porous PEEK samples were impregnated in $2 \mathrm{mg} / \mathrm{mL}$ dopamine (Sigma-Aldrich) in Tris- $\mathrm{HCl}$ solution $(10 \mathrm{mM}, \mathrm{pH} 8.5)$ with vibration at $37^{\circ} \mathrm{C}$ for $24 \mathrm{~h}$ to yield the first layer of pDA through a spontaneous polymerization of dopamine, followed by an ultrasonic wash in D.I. water to remove excess monomer and particles. Subsequently, the samples were subjected to $\mathrm{AgNO}_{3}$ solution $(1 \mathrm{mM})$ for $8 \mathrm{~h}$, and then were treated by ultraviolet ( $\mathrm{UV}, \lambda=254 \mathrm{~nm}, 5 \mathrm{~W} / \mathrm{m}^{2}$ ) for another $1 \mathrm{~h}$ to further reduce $\mathrm{Ag}^{+}$. Secondly, the Ag-decorated PEEK scaffolds (3P-Ag) further received a second layer of pDA following the same pDA decoration procedure. Afterward, the scaffolds were immersed in a 1.5X SBF solution for 2 days for apatite anchoring. The resulting scaffolds were rinsed out using D.I. water to remove unfixed apatite and were named as 3P-Ag-AP; (2) 3P-AP-Ag scaffolds: As the name suggested, the 3P-AP-Ag scaffolds were fabricated using the same dual pDA-assisted method, but the difference resides in the biofunctionalization sequence of apatite and AgNPs. The 3D-printed PEEK scaffolds firstly underwent apatite deposition using SBF solution, followed by $\mathrm{AgNO}_{3}$ immersion and UV reduction.

The bare 3P scaffolds without any treatment were also prepared as a control group. Besides, the AgNPs-decorated scaffolds (3P-Ag) and apatite-decorated scaffolds (3P-AP) were prepared by immersing the pDA-modified PEEK scaffolds into $\mathrm{AgNO}_{3}$ solution and SBF solution, respectively, for comparison in this work. The schematic illustration of the proposed coatings is shown in Figure 1a.

\section{Material Characterization}


We used a field-emission scanning electron microscope (FE-SEM, JEOL, JSM-6701F, Japan) equipped with energy dispersive spectrometer (EDS) to analyze the surface microstructures and chemical elements of coatings. X-ray photoelectron spectroscopy (XPS, Kratos, UK) has been applied to detect the chemical components. The hydrophilicity was investigated through a water contact angle (CA) goniometry apparatus (SL200B, Kino, USA) equipped with a high-resolution camera. The mechanical properties of the as-prepared 3D-printed PEEK scaffolds were carried out using a universal material testing machine (AG-IC 50 kN, Shimadzu, Japan).

\section{Multiple Ions Delivery in Distinct pH of PBS}

To probe the delivery profiles of $\mathrm{Ag}^{+}$and $\mathrm{Ca}^{2+}$ ions from coatings, specimens $(n=6)$ were immersed in $3 \mathrm{~mL}$ of PBS buffer with distinct $\mathrm{pH}$ values $(\mathrm{pH}=5.0,6.0,7.4)$ at $37^{\circ} \mathrm{C}$ under static condition for consecutive 28 days (d). The leachate was collected at a preplanned time ( 1 h, $3 \mathrm{~h}, 6 \mathrm{~h}, 12 \mathrm{~h}, 1 \mathrm{~d}, 3 \mathrm{~d}, 5 \mathrm{~d}, 7 \mathrm{~d}, 10 \mathrm{~d}, 14 \mathrm{~d}, 21 \mathrm{~d}, 28 \mathrm{~d})$, and then refilled with new PBS accordingly. The analysis was measured through an inductively coupled plasma mass spectrometry (ICP-MS, Leeman, USA). Besides, the total Ag contents on different modified PEEK scaffolds were also tested by immersing them in $\mathrm{HNO}_{3}(n=6)$.

\section{Antibacterial Assessments}

\section{Bacterial Culture}

Two common bacteria, E. coli (ATCC 25925) and S. aureus (ATCC 25924), were applied to evaluate antibacterial performances of these bioscaffolds. The bacteria were multiplied in liquid Luria-Bertani (LB) media under the aerobic humid condition at 
$37{ }^{\circ} \mathrm{C}$. After growing overnight, the concentrations of bacterial suspensions were diluted to desirable concentrations for ensuing experiments.

\section{Inhibition Zone Tests}

To conduct the inhibition zone antibacterial assessment, the aseptic scaffolds $(n=6)$ were placed at the center of the LB agar plates, which has been evenly coated with $200 \mu \mathrm{L}$ of two bacterial suspensions with a concentration of $1 \times 10^{6} \mathrm{CFU} / \mathrm{mL}$. The bactericidal action of the bioscaffolds was evaluated as the size of the bacterial growth inhibition zone after cultivation for $24 \mathrm{~h}$ at $37^{\circ} \mathrm{C}$.

\section{Antibacterial Growth Curves}

All 3D printed scaffolds were sterilized through autoclaved sterilization method. The aseptic samples were put in a test tube, covered with $40 \mu \mathrm{L}$ of two types of bacterial suspension and $2 \mathrm{~mL} \mathrm{LB}$ media, and they were cultivated at standard temperature. At different culturing time points, the absorbance value (OD value) of the media with the wavenumber of $600 \mathrm{~nm}$ was detected with a microplate reader (SAF-680T, BAIIU, China).

\section{SEM Observation of Bacterial Morphology}

Scaffolds for bacterial integrity observation were placed into $500 \mu \mathrm{L}$ of suspension with a concentration of $1 \times 10^{6} \mathrm{CFU} / \mathrm{mL}$ bacteria and cultured for $24 \mathrm{~h}$. Afterward, specimens were rinsed with PBS and fixed by $2.5 \%$ glutaraldehyde for $4 \mathrm{~h}$. Scaffolds were serially dehydrated in gradient ethanol (30-100\%, 20 min for each step), and dried using a critical point dryer. The morphology and integrity of bacteria were observed using an FE-SEM (JSM-6701F). 


\section{Cell Experiments}

\section{Cell Culture and Seeding}

Pre-osteoblastic MC3T3-E1 cells, obtained from ATCC, were cultivated in Dulbecco's modified Eagle's medium (DMEM) (KeyGEN BioTECH, Nanjing) supplemented with $10 \%$ fetal bovine serum (FBS, Hyclone, USA) and $1 \%$ penicillin-streptomycin (Gibco) in a humidified standard cell culture condition $\left(37^{\circ} \mathrm{C}\right)$. All 3D printed scaffolds were sterilized through autoclaved sterilization method. The disinfected scaffolds were placed in 48-well tissue culture plastic (Corning, USA) with a cell density of $4 \times 10^{4} /$ well. The cell media was changed every 2-3 days. For all cell experiments, six parallel samples were performed in triplicate to obtain statistically acceptable results.

\section{Cell Proliferation}

After cells had been incubated with the scaffolds for 1, 3, and 5 days, the commercial Cell Count Kit (CCK-8, Dojindo, Japan) was added to the cells at a proportion of 1:10 for reaction with cells for $3 \mathrm{~h}$. Subsequently, the absorbance of each $100 \mu \mathrm{L}$ supernate from different groups was recorded at $450 \mathrm{~nm}$ using 96 -well plates by the microplate reader $(\mathrm{SAF}-680 \mathrm{~T})$.

\section{Morphology and Cytoskeleton of Cells}

The morphologies of MC3T3-E1 cells grown on the 3D scaffolds were captured using the FE-SEM. After $24 \mathrm{~h}$ culture, scaffolds with cells were rinsed with PBS and fixed by $2.5 \%$ glutaraldehyde. Then cells were serially dehydrated using gradient series ethanol, dried, and finally sputter-coated by gold for SEM observation (JSM-6701F). 
At 2 days of culture, cells were rinsed by PBS buffer and fixed with $4 \%$ paraformaldehyde for 40 min. After being permeabilized with $0.1 \%$ Triton X-100 (Sigma-Aldrich) for $8 \mathrm{~min}$, osteoblasts were incubated in $0.5 \% \mathrm{BSA} / \mathrm{PBS}$ solution for $0.5 \mathrm{~h}$ to block non-specific binding, followed by introducing $5 \mu \mathrm{g} / \mathrm{mL}$ TRITC-labeled phalloidin (Sigma-Aldrich) for F-action staining for $40 \mathrm{~min}$. After rinsing with PBS, cells were further reacted with $8 \mu \mathrm{g} / \mathrm{mL}$ DAPI (Sigma-Aldrich) for $5 \mathrm{~min}$. The stained signals were observed using a confocal laser scanning microscopy (CLSM, LSM510, Carl Zeiss, Germany).

\section{Osteogenic Capacity Studies}

\section{Alkaline Phosphate (ALP) Activity}

Intracellular release of ALP activity was determined at 7 and 14 days by a commercial ALP assay kit (Nanjing Jiancheng Bioengineering) according to the manufacture's guidelines. ${ }^{3}$ The total protein contents were evaluated by a Bicinchoninic Acid (BCA) protein assay kit (Thermo, USA) to normalize the results. Ultimately, ALP activity was determined as the total protein content (U/gprot). Meanwhile, the visualized ALP distribution and expression on scaffolds were also evaluated using a BCIP/NBT ALP staining kit (Beyotime).

\section{Calcium Mineralization}

The deposition of calcium nodules was assayed at 21 days by Alizarin Red S (ARS) staining (Sigma). Briefly, cell layers on scaffolds were fixed and stained by ARS solution ( $3 \%, \mathrm{pH} 4.5)$ for $1 \mathrm{~h}$, and then the excess ARS was washed with D.I. water at least five times. Besides, samples without cells (as a control group) were also stained 
using the same method to avoid the interference of calcium originally on the materials. The stained bioscaffolds were imaged by a scanner. Quantitatively, the ARS dyes on samples were eluted overnight with the assistance of $10 \%$ hexadecylpyridinium chloride aqueous solution (Sigma-Aldrich). The measurements were conducted at an OD value of $450 \mathrm{~nm}$ using the microplate reader (SAF-680T). Finally, the quantitative data needs to exclude the background interference.

\section{Expression of Osteogenesis-Specific Genes}

In order to evaluate the osteogenic potential of cells on the studied scaffolds at the molecular level, the total mRNA was firstly isolated using TRIzol (Invitrogen, USA) and converted into cDNA using a RevertAid First Strand cDNA Synthesis Kit (Thermo). A quantitative real-time polymerase chain reaction (RT-qPCR) was conducted using an ABI 7500 Realtime-PCR machine (Applied Biosystems, USA) using a thermal process in line with our previous studies. ${ }^{4,5}$ Each group was run in triplicate, and GAPDH was assigned as the endogenous control. Primers of these osteogenesis-specific genes were provided in Table S3. Ultimately, the mRNA folds were determined according to the standard $\Delta \Delta \mathrm{Ct}$ method.

\section{In Vivo Tissue Integration Evaluation}

\section{Infective Bone Defect Models in Rats}

Animal surgery and experiments were authorized by the Institutional Animal Care and Use Committee at the Sichuan University. Forty 6-week-old female Sprague Dawley (SD) rats were used for the construction of an infective critical-sized calvarial defect model. The surgical procedures were described in our previous work. ${ }^{6}$ 
In brief, the rats were anesthetized by pentobarbital sodium $(3 \%, 40 \mathrm{mg} / \mathrm{kg})$ through intraperitoneal injection. As a first step, critical-sized defects ( $8 \mathrm{~mm}$ in diameter) were created in the middle of the skull by a dental trephine. The rats were divided into four groups based on the implanted scaffolds: 3P $(n=10), 3 \mathrm{P}-\mathrm{Ag}(n=10), 3 \mathrm{P}-\mathrm{AP}(n=10)$, and 3P-Ag-AP scaffolds $(n=10)$. These scaffolds were contaminated using S. aureus in $1 \mathrm{~mL}$ PBS $\left(1 \times 10^{6} \mathrm{CFU} / \mathrm{mL}^{-1}\right)$ for $0.5 \mathrm{~h}$ to permit the bacteria to adhere to the surface of porous implants. Then, the bacteria-contaminated implants were implanted into the defect region, matching the dimension of the implants. After suture, the rats were fed normally, and no antibiotics were administered post-operatively. At 5 weeks after the operation, these rats were sacrificed via euthanasia, and their calvaria was harvested, fixed in $10 \%$ formalin solution for further evaluations.

\section{In Vivo Antibacterial Evaluation}

The in Vivo antibacterial efficiency was evaluated by the spread plate approach. Briefly, after sacrifice, the retrieved scaffolds were immediately collected and placed in an ultrasonic bath with $5 \mathrm{~mL}$ PBS for $5 \mathrm{~min}$ to detach adhered bacteria from scaffolds surface. Such bacteria-containing PBS was diluted 10 folds and uniformly spread onto the LB agar for 1-day incubation to calculate the number of viable bacteria from different scaffolds.

\section{Histological Evaluation by Undecalcified Slices}

After graded dehydration in ethanol series (40-100\%), the undecalcified calvaria samples containing implantable scaffolds ( $n=5$ for each group) were immersed in methyl methacrylate (MMA) allowing for polymerization for about 2 months. Then, 
the well-embedded specimens were longitudinally cut to section with a thickness of about $30 \mu \mathrm{m}$ (Leica SP1600, Germany). The undecalcified sections were treated with Van Gieson staining to analyze the newly-formed bony tissues and bone in-growth inside the implants.

\section{Histological and Immunohistochemical Evaluations by Decalcified Slices}

The remaining skull samples were exposed to $15 \%$ EDTA solution for 3 weeks. After dehydration in gradient ethanol solutions, samples were embedded in paraffin after the internal implants were carefully removed. The histological sections (108 $\mu \mathrm{m})$ around the implanted scaffolds from each group were prepared for H\&E, Masson' trichrome, and immunohistochemical staining, including osteogenesis-specific proteins (OCN and OPN) and angiogenesis-specific proteins (VEGF and VWF). This immunohistochemical staining was conducted according to the manufacturers' instructions. $^{7,8}$

\section{Statistical Analysis}

All data were expressed as means \pm standard deviations (SD), and the statistical analysis was conducted using one-way variance analysis (ANOVA), wherein $p<0.05$ was considered statistically significant.

\section{REFERENCES}

(1). Ouyang, L.; Deng, Y.; Yang, L.; Shi, X.; Dong, T.; Tai, Y.; Yang, W.; Chen, Z.-G. Graphene-Oxide-Decorated Microporous Polyetheretherketone With Superior Antibacterial Capability and In Vitro Osteogenesis for Orthopedic implant. Macromol. 
Biosci. 2018, 18, 1800036.

(2) Deng, Y.; Zhou, P.; Liu, X.; Wang. L.; Xiong, X.; Tang, Z.; Wei, J.; Wei, S. Preparation, Characterization, Cellular Response and In Vivo Osseointegration of Polyetheretherketone/Nano-Hydroxyapatite/Carbon Fiber Ternary Biocomposite. Colloids Surf. B Interfaces 2015, 136, 64-73.

(3) Jia, Z.; Xiu, P.; Roohani-Esfahani, S.-I., Zreiqat, H.; Xiong, P.; Zhou, W.; Yan, J.; Chen, Y.; Zheng.; Y. Triple-Bioinspired Burying/Crosslinking Interfacial Coassembly Strategy for Layer-by-Layer Construction of Robust Functional Bioceramic Self-Coatings for Osteointegration Applications. ACS Appl. Mater. Interfaces 2019, 11, 4447-4469.

(4) Deng, Y.; Wei, S.; Yang, L.; Yang, W.; Dargusch, M. S.; Chen, Z.-G. A Novel Hydrogel Surface Grafted With Dual Functional Peptides for Sustaining Long-Term Self-Renewal of Human Induced Pluripotent Stem Cells and Manipulating Their Osteoblastic Maturation. Adv. Funct. Mater. 2018, 28, 1705546.

(5) Deng, Y.; Yang, Y.; Wei, S. Peptide-Decorated Nanofibrous Niche Augments In Vitro Directed Osteogenic Conversion of Human Pluripotent Stem Cells. Biomacromolecules 2017, 18, 587-598.

(6). Deng, L.; He, X.; Xie, K.; Xie, L.; Deng, Y. Dual Therapy Coating on Micro/Nanoscale Porous Polyetheretherketone to Eradicate Biofilms and Accelerate Bone Tissue Repair. Macromol. Biosci. 2019, 19, 1800376.

(7) Yang, Y.; Yang, S.; Wang, Y.; Yu, Z.; Ao, H.; Zhang, H.; Qin, L.; Guillaume, O.; Eglin, D.; Richards, R. G.; Tang, T. Anti-Infective Efficacy, Cytocompatibility and 
Biocompatibility of a 3D-Printed Osteoconductive Composite Scaffold Functionalized With Quaternized Chitosan. Acta Biomater. 2016, 46, 112-128.

(8) Yang, Y.; Chu, L.; Yang. S.; Zhang, H.; Qin, L.; Guillaume, O.; Eglin, D.; Richards, R. G.; Tang, T. Dual-Functional 3D-Printed Composite Scaffold for Inhibiting Bacterial Infection and Promoting Bone Regeneration in Infected Bone Defect Models. Acta Biomater. 2018, 79, 265-275. 
Supporting Images and Tables

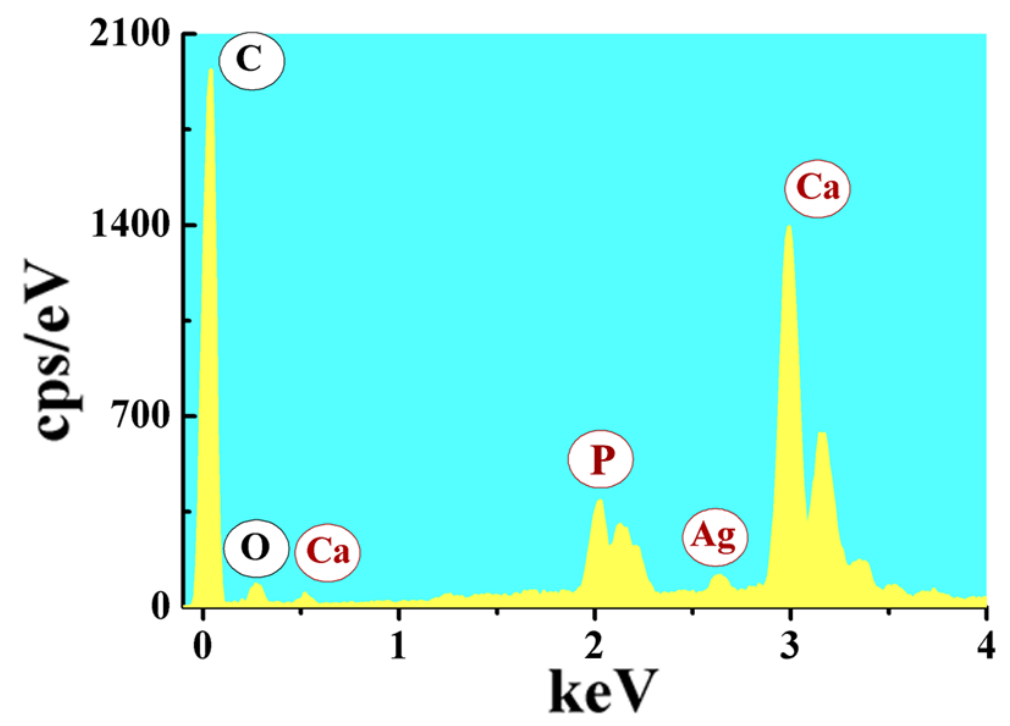

Figure S1. The EDS point sweep corresponding to the arrow of Figure 2e showing C, $\mathrm{O}, \mathrm{Ca}, \mathrm{P}$, and Ag elements on the surface of 3P-AP-Ag scaffolds. 

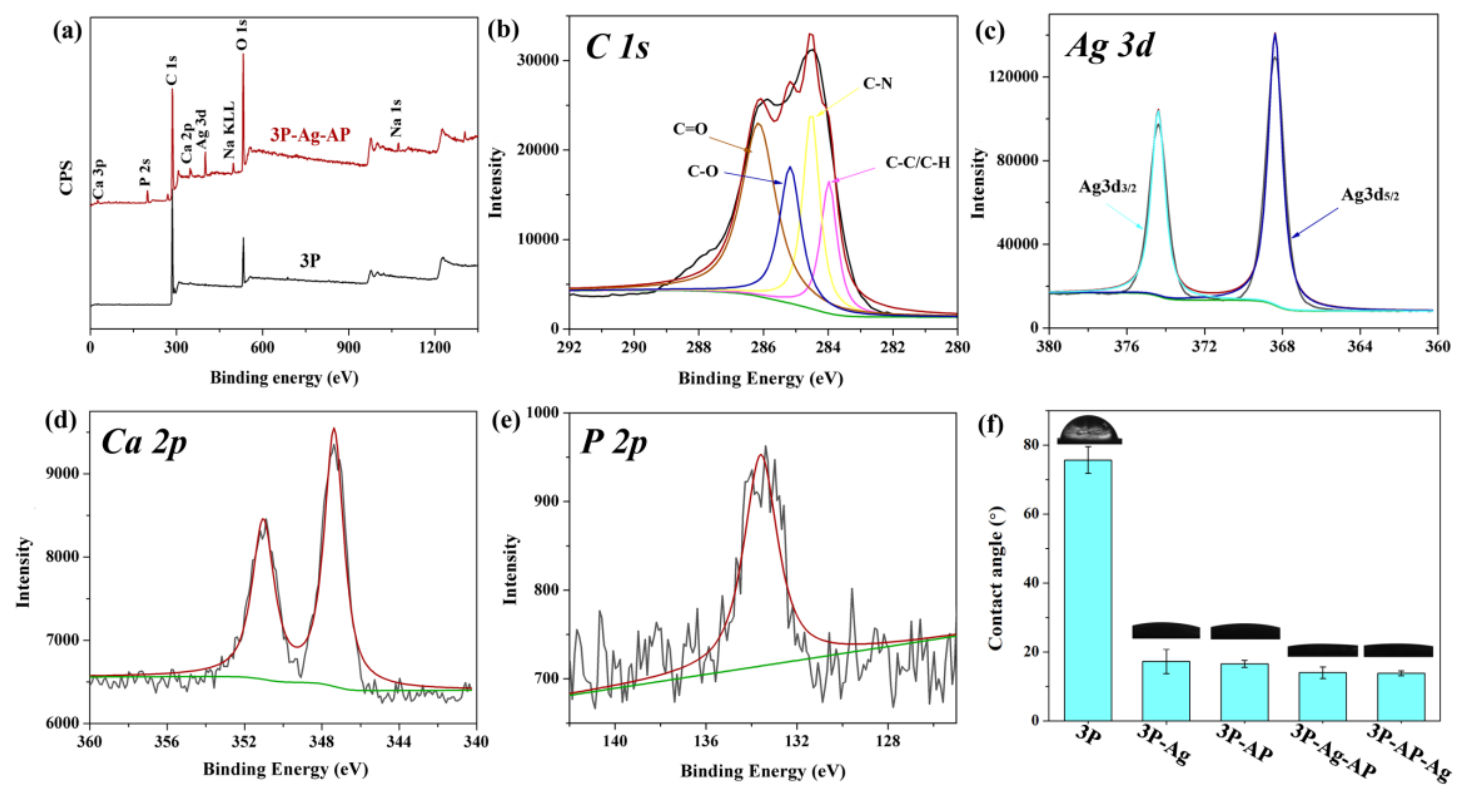

Figure S2. Chemical composition and contact water evaluation: (a) XPS survey spectra of 3P and 3P-Ag-AP; High-resolution spectra of (b) C 1s, (c) Ag 3d, (d) Ca $2 p$ and (e) P $2 p$ for 3P-Ag-AP; and (f) Hydrophilicity of these multifunctional scaffolds. 

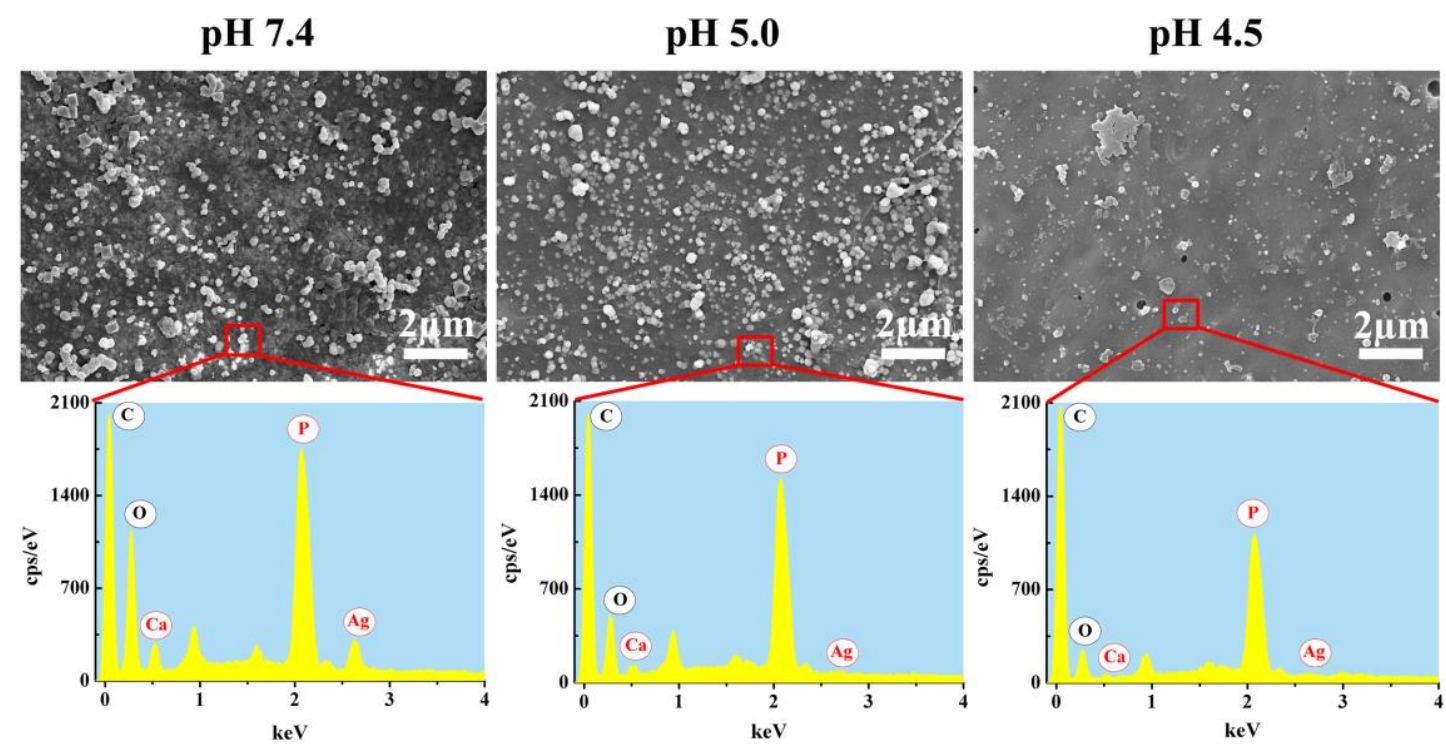

Figure S3. Surface morphology and corresponding EDS of 3P-Ag-AP samples after treatment with different $\mathrm{pH}$ for 28 days immersion in PBS. 

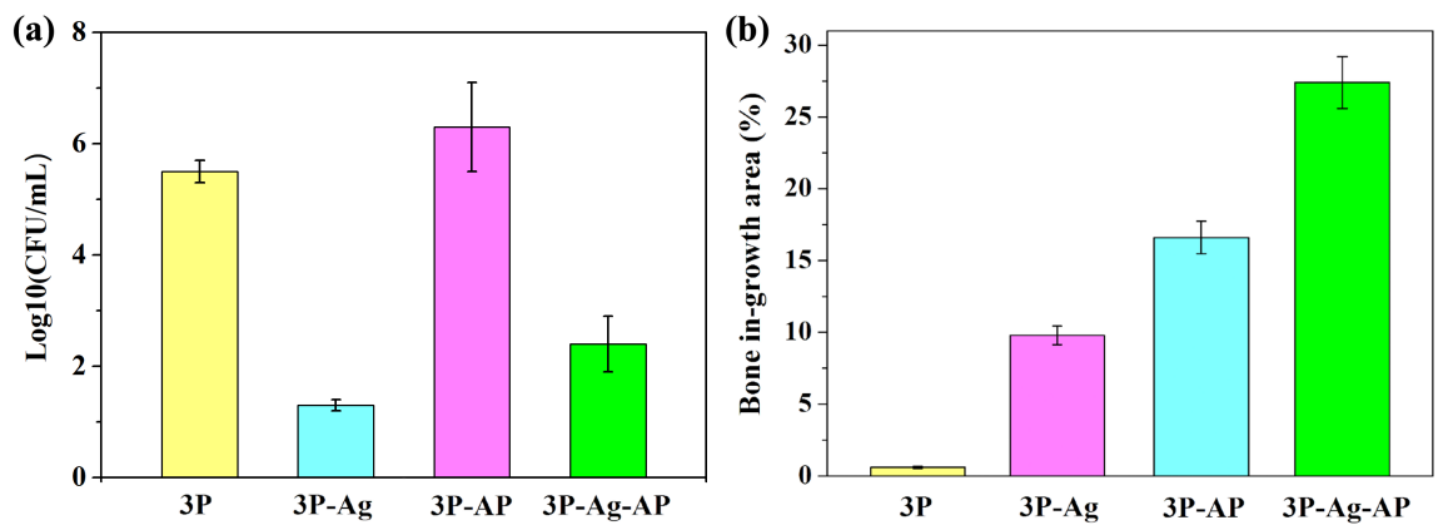

Figure S4. (a) The quantitative statistical analysis of the viable bacteria on different

PEEK scaffold implants; (b) Quantitative analysis of the new-regenerated bone region stained from Van Gieson staining. $(n=6)$ 
Table S1. The total loading amount of Ag on different modified PEEK scaffolds determined by ICP-MS.

\begin{tabular}{cccc}
\hline Sample & 3P-Ag & 3P-Ag-AP & 3P-AP-Ag \\
\hline Ag content & $3.26 \pm 0.11$ & $2.82 \pm 0.18$ & $4.37 \pm 0.25$ \\
& $\mu \mathrm{g} / \mathrm{mg}$ scaffold & $\mu \mathrm{g} / \mathrm{mg}$ scaffold & $\mu \mathrm{g} / \mathrm{mg}$ scaffold \\
\hline
\end{tabular}


Table S2. Ion concentrations of 1.5X SBF (mM).

\begin{tabular}{cccccccc}
\hline $\mathrm{Na}^{+}$ & $\mathrm{K}^{+}$ & $\mathrm{Mg}^{2+}$ & $\mathrm{Ca}^{2+}$ & $\mathrm{Cl}^{-}$ & $\mathrm{HCO}_{3}{ }^{-}$ & $\mathrm{HPO}_{4}{ }^{2-}$ & $\mathrm{SO}_{4}{ }^{2-}$ \\
\hline 213.0 & 7.5 & 2.3 & 3.8 & 221.7 & 6.3 & 1.5 & 0.8 \\
\hline
\end{tabular}


Table S3. Sequences of primer pairs used in RT-qPCR analysis.

\begin{tabular}{ccc}
\hline Gene & Forward primer & Reverse primer \\
\hline ALP & CCAACTCTTTTGTGCCAGAGA & GGCTACATTGGTGTTGAGCTTTT \\
Runx2 & AGGAATGCGCCCTAAATCACT & ACCCAGAAGGCACAGACAGAAG \\
CO11a1 & GCTCCTCTTAGGGGCCACT & CCACGTCTCACCATTGGGG \\
OCN & GAACAGACAAGTCCCACACAGC & TCAGCAGAGTGAGCAGAAAGAT \\
GAPDH & GGTGAAGGTCGGTGTGAACG & CTCGCTCCTGGAAGATGGTG \\
\hline
\end{tabular}

